\title{
Lumbosacral Corsets Improve the Outcome of Patients with Non-Specific Acute Low Back Pain
}

\author{
Aruyaru Stanley Mwenda ${ }^{1}$, Akil Fazal ${ }^{1}$, Hassan Saidi ${ }^{1},{ }^{2}$, Parmenas Oroko $^{1}$ \\ 1. Aga Khan University Hospital, Nairobi \\ 2. University of Nairobi
}

Correspondence to: Dr Stanley Aruyaru, P.0 Box 25-10100 Nyeri, Kenya. arumwesta@gmail.com.

\begin{abstract}
Introduction: Low back pain (LBP) is a common global problem affecting $60-90 \%$ of the population in their lifetime. It is a leading reason for hospital admission, healthcare spending, workplace absenteeism and years lived with disability. Up to $90 \%$ of patients suffering from low back pain do not have a serious pathology warranting imaging or further intervention. These are categorized as having non- specific low back pain. This has a good prognosis running a short course of 3 to 6 weeks. The use of corset as a physical therapy for non-specific acute LBP has not been clearly supported with scientific evidence. However, it is commonly prescribed for this category of patients. It is important to assess the effect of this modality on the outcome of patients with non- specific acute LBP. Methods: This study aimed to assess the outcome of patients with non-specific acute low back pain treated with a corset in addition to a standard analgesic protocol compared to those on a standard analgesic protocol alone over 3- week duration at the Aga Khan University Hospital, Nairobi, Kenya. Results: Seventy-nine patients were analyzed. There was a significant effect of time on the
\end{abstract}

\section{Introduction}

Low back pain (LBP) is a common global cause of morbidity (1). It is a leading cause of hospital admission and healthcare spending $(1,2)$. It is estimated that up to $60-90 \%$ of the general population will develop low back pain in their lifetime $(1,2)$. Because of this magnitude of disease, LBP is an important cause of work place absenteeism and a leading consumer of healthcare funding $(1,2)$. It is estimated to cost over
Oswestry Disability Index (ODI) $(\mathrm{F}=(1.38,106.56)$ $=207.89, \mathrm{P}=.000$ ). There was a statistically significant difference in ODI score between the two arms favoring the intervention $\operatorname{arm}(\mathrm{F}(1,27)=4.23, \mathrm{p}=.043)$. The difference in pain score and days off duty was not statistically significant. The number of days off duty was comparable between the two arms. Compliance to use of corset was good with mean of 1 day off the corset. The maximal change in both ODI and pain score occurred between the first and second reviews indicating maximum clinical improvement within the first week. Conclusion: Compared to use of analgesia alone, the use of additional corset among patients with non-specific acute LBP resulted in a significant improvement in their back specific disability

Key words: Non-Specific Low Back Pain, Lumbosacral Corset, Disability

Ann Afr Surg. 2017;14(1): 35-39.

DOI: http://dx.doi.org/10.4314/aas.v14i1.7

(C) 2017 Annals of African Surgery. This work is licensed under the Creative Commons Attribution 4.0 International License.
149 million lost work days and 100 to 200 billion dollars per year in the United States (3). As a result, early symptom relief and resumption of daily activity is always a priority. Management of non-specific acute low back pain (NSALBP) entails pharmacological and non- pharmacological interventions for pain control and back rehabilitation with the aim of rapid return to normal activity (1)

Lumbosacral corset (also known as support or brace) 
as a form of physical therapy for acute low back has been used with varying results (1-3). Potential benefits of lumbosacral corsets include stabilization and reduction of spine motion, reduction of mechanical loading; and provision of the miscellaneous effects of massage, heat and as a placebo $(1,4,5)$. They are also thought to protect the spine through avoiding extreme trunk motion and offering tactile biofeedback (5). There is paucity of data on the use of lumbosacral corset for acute low back pain. Existing literature on the use of lumbosacral corset is deficient of high quality randomized trials assessing the importance of this modality of treatment on acute low back pain with regard to pain relief and disability improvement (6). No studies exist locally to shed light on the same. The decision to use or not use a corset is made on a physician by physician basis; sometimes dependent on the patient request or the physiotherapist input. A study assessing the impact of additional lumbosacral corset to other modes of therapy should, besides adding to the body of knowledge; form evidence based reference locally and guide the choice of intervention for patients with NSALBP. To the authors' knowledge, this is the first study in English literature addressing the use of lumbosacral corset in patients with NSALBP.

\section{Methods}

This study aimed to assess the outcome (change in back specific disability) of patients with non-specific acute low back pain treated with a lumbosacral corset in addition to a standard analgesic protocol compared to those on a standard analgesic protocol alone over 3- week duration at the Aga Khan University Hospital, Nairobi, Kenya. The secondary objectives were to measure the change in pain score, quantify days off duty due to back pain, describe duration to maximal change in pain and disability and to assess the patients' compliance to use of a lumbosacral corset.

Eighty two patients were randomly assigned to either corset or no corset arms by picking opaque envelopes. The primary outcome was the change in back specific disability as measured on the Oswestry Disability Index (ODI). Secondary outcomes included pain as assessed by Visual Analogue Score (VAS), number of days off duty and number of days off analgesia. The patients were followed up weekly for three weeks. At each point their ODI score was calculated and recorded, as were other secondary outcomes. The research protocol has been submitted separately for publication elsewhere.

\section{Results}

A total of 90 patients were assessed for eligibility, out of which 82 were recruited into the study. Three subjects ( 2 intervention, 1 control) were lost to follow up leaving 79 patients for final analysis of the primary outcome (38 Intervention, 41 control). Four patients required admission for one day (3 intervention and 1 control). Of these four, one patient (control arm) was admitted at the end of the first week due to persistent pain and given parenteral analgesics, while 1 patient (intervention arm) required MRI scan due to worsening pain. She was found to have nerve root compression from a prolapsed intervertebral disc and she underwent microdiscectomy.

Baseline characteristics of the control and intervention groups were comparable. Sex and occupation were also similar between groups $(\mathrm{p}=0.363$ and 0.643 respectively) (Table 1).

Table 1: Baseline characteristics at Start of the study

\begin{tabular}{|l|c|c|c|}
\hline & Control & Intervention & Sig \\
\cline { 2 - 4 } & $\mathrm{n}=42$ & $\mathrm{n}=40$ & $p$ \\
\hline Age & 40.64 & 40.55 & 0.238 \\
\hline ODI & 34.64 & 41.20 & 0.421 \\
\hline VAS & 5.81 & 6.18 & 0.539 \\
\hline
\end{tabular}

The results show that there was a significant effect of time on the ODI, $\mathrm{F}=(1.38,106.56)=207.89, \mathrm{p}=.000$. These results suggest that with the standard treatment protocol, with or without the lumbosacral corset, ODI improved over time. Between-subject effects showed a significant difference between the groups, (F (1, 77) $=4.23, p=0.043$ ) suggesting that patients in the intervention group had a greater improvement in ODI over the study period. (Table 2, Figure 1)

Table 2: Days off duty, analgesia in both Groups

\begin{tabular}{|l|l|l|l|l|}
\hline & & N & Mean & SD \\
\hline Days off Work & Intervention & 30 & 1.93 & 3.552 \\
\hline & Control & 38 & 1.95 & 3.058 \\
\hline & & & & \\
\hline Days offAnalgesia & Intervention & 8 & 1.38 & 1.685 \\
\hline & Control & 14 & 0.79 & 1.311 \\
\hline
\end{tabular}




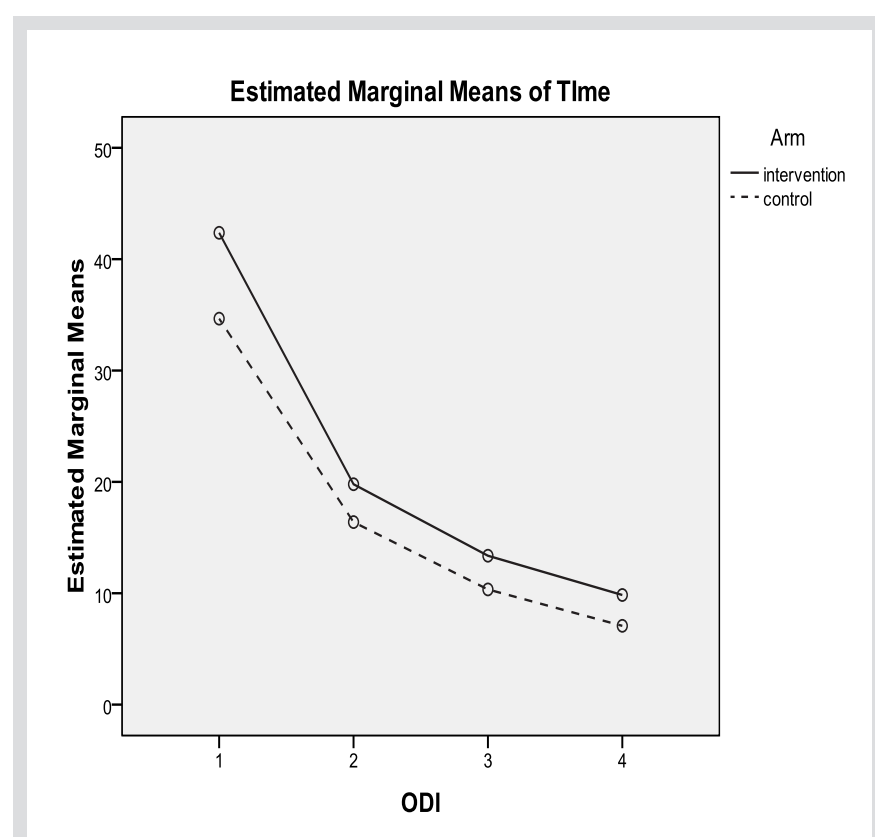

Figure 1: Change in mean ODI over time

Mauchlys' test indicated sphericity did not hold, $(\chi 2$ $(5)=64.03, p=.000)$. Correction with GreenhouseGeisser correction $(\varepsilon=0.461)$ revealed a significant effect of time on the Visual Analog Score for pain, $(\mathrm{F}=(1.98,152.39)=381.21, \mathrm{p}=.000)$, suggesting an improvement in the VAS with time among subjects in both arms. There was however no significant difference between the control and intervention arms ( $F(1,78)=0.189, \mathrm{p}=0.665)$. (Figure 2$)$.

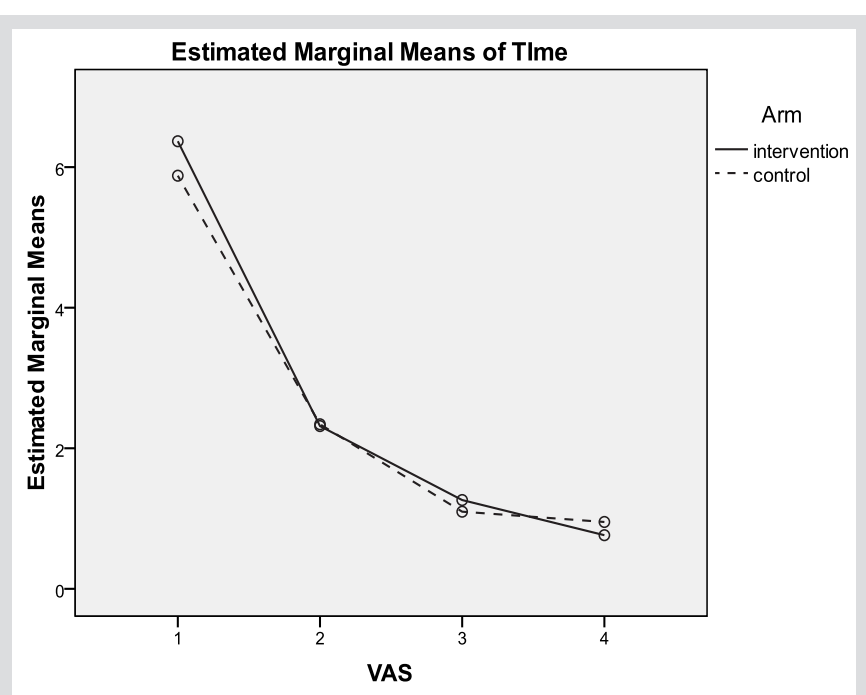

Figure 2: Mean change in VAS over time

There was no statistical difference in days off duty from work due to back pain between the control and intervention groups $(\mathrm{F} 1=.000, \mathrm{p}=.986)$. These data are summarized in Table 2.

Days off analgesia were calculated for the first one week. Data was complete for $22 / 79$ patients. No statistical difference was detected between the arms (F1 = .837, $\mathrm{p}=.371$ ) (Table 2). Change in disability (ODI) and pain scores were similar between the intervention and control groups as summarized in Table 3. Mean duration of time off corset was 0.76 days (SD 0.863) $(n=37)$. This was not significantly correlated with sex, $\mathrm{F}(1,36)=0.269, \mathrm{p}=.607$ nor age, $\mathrm{F}(1,36)=3.76, \mathrm{p}=.061$.

Table 3: Initial and maximal change in VAS and ODI

\begin{tabular}{|c|c|c|c|c|c|c|c|}
\hline & & $\mathrm{N}$ & Mean & SD & df $(x, 77)$ & $\mathrm{F}$ & $\mathrm{P}$ \\
\hline \multirow{2}{*}{ Max Response ODI } & intervention & 38 & 31.68 & 14.93 & \multirow[t]{2}{*}{1} & \multirow[t]{2}{*}{1.235} & \multirow[t]{2}{*}{.270} \\
\hline & control & 41 & 27.63 & 17.26 & & & \\
\hline \multirow{2}{*}{$\begin{array}{l}\text { Max Response } \\
\text { VAS }\end{array}$} & intervention & 38 & 5.63 & 1.85 & \multirow[t]{2}{*}{1} & \multirow[t]{2}{*}{3.169} & \multirow[t]{2}{*}{.079} \\
\hline & control & 41 & 4.85 & 2.02 & & & \\
\hline \multirow{2}{*}{$\begin{array}{l}\text { Initial Response } \\
\text { ODI }\end{array}$} & intervention & 38 & 20.89 & 11.94 & \multirow[t]{2}{*}{1} & \multirow[t]{2}{*}{2.044} & \multirow[t]{2}{*}{.157} \\
\hline & control & 41 & 16.80 & 13.37 & & & \\
\hline \multirow{2}{*}{$\begin{array}{l}\text { Initial Response } \\
\text { VAS }\end{array}$} & intervention & 38 & 4.05 & 1.82 & \multirow[t]{2}{*}{1} & \multirow[t]{2}{*}{1.555} & \multirow[t]{2}{*}{.216} \\
\hline & control & 41 & 3.54 & 1.86 & & & \\
\hline
\end{tabular}




\section{Discussion}

This study established a positive correlation between change in both ODI and VAS and time, such that both the back specific disability and the pain score reduced over time in both arms. This was statistically significant. Our findings are in agreement with other authors who have observed that $90 \%$ of patients with NSALBP will heal within six weeks (7). In fact Calmels et al argue that in some cases $50 \%$ of patients with NSALBP will recover in one week and $90 \%$ within two weeks (1). In a randomized clinical trial involving patients with all classes of LBP, Valle-Jones et al evaluated pain control, analgesic requirements and resumption of duty among patients treated either with analgesics alone or those with additional lumbosacral corset over 21 days and demonstrated a similar trend (8). This shows that in general, Non specific LBP resolves quickly over time.

For the primary outcome, this study found that the use of the lumbosacral corset in addition to analgesic protocol was associated with a statistically significant improvement in back specific disability as assesed via the ODI. Other researchers have demonstrated better outcome using various tools on different subsets of patients with LBP $(1,8)$. In a multicentre randomized trial, Calmels and colleagues found that the use of elastic lumbar belts among patients with sub acute LBP significantly reduced the pain score, analgesic requirement and back disability over a 90 day duration (1). The duration of follow-up for Valle-Jones et al trial was 21 days similar to ours. They too demonstrated earlier resumption of work in the lumbosacral corset arm but they did not use any back specific disability scoring tool (8). Their sample included patients with acute, sub acute and chronic LBP. Ours was the first study to investigate this on patients with NSALBP only. Different possible mechanisms by which lumbosacral corsets act have been postulated (5). It is not possible to describe how the corset in this study acted to improve the disability outcomes, and whether one or all mechanisms could be applicable. As stated earlier, it would be hypothesized that the effect of corset may have been by reduction of extreme trunk motion via tactile biofeedback $(5,9)$. It is also possible that the presence of a lumbosacral corset gave the patients the courage to initiate back activity earlier compared to the control arm patients who might have feared to worsen their pain and hence limited their back activity. It would be interesting to find out if there is a specific domain on the ODI tool that persistently changed more in the intervention arm to sway the results of the cumulative ODI score.

Regarding the secondary outcome of change in pain score, there was no statistically significant difference between the arms. In the same setting and using a similar methodology, Mohammedali et al did not demonstrate any difference in pain score between NSALBP patients treated with analgesics alone compared to those treated with additional ultrasound therapy (10). This means thelumbosacral corset did not contribute to pain reduction in our study population. This is in contradistinction to the ODI score. Pain severity is the first component of the 10-section ODI tool. In fact all the other 9 sections assessing various domains of back related disability are constructed assuming that pain is the limiting factor (11). Whether it is actually pain itself or the fear of anticipated pain that limits patients' activity along these 9 domains is not easy to establish. The findings of our study may indicate that it is the fear of hurting one's back that would limit their activity, and not the back pain itself. Thus once the pain was relieved by analgesia, the patients with a corset may have felt more confident to engage in activities that required the use of their back while those on analgesics alone were still cautious even with good pain control. It could as well be from various other mechanisms that have been postulated to explain the effects of lumbosacral corsets $(5,9,11)$. Regarding days off duty, data was available for 68/79 patients analyzed (86\%). The days \{mean, (SD)\} off duty were 1.93 (3.5) and 1.9 (3.05) for the intervention and control arms respectively. The difference was not statistically significant $(\mathrm{F} 1=.837, \mathrm{p}=.371)$. This means that NSALBP kept patients off duty on average for two days. This concurs with our earlier finding of improvement over time in both back specific disability and pain score, indicating that patients recovered quickly enough to be able to resume duties. As highlighted by other authors, NSALBP generally runs a favorable prognosis with patients improving as early as one week $(1,7,8)$. Our study was not however powered to detect differences in days off duty and thus we cannot draw strong conclusions in this regard. The maximal change in pain score and ODI occurred after a week of treatment. There was no statistically significant difference between the two arms. This means that irrespective of the arm, the treatment initiated brought about a clinical improvement. Mohammedali et al demonstrated the same finding in the same setting (10). The study was however not powered to assess these secondary outcomes so strong conclusions cannot be drawn.

Compliance to the use of the corset was good with an average of 1 day off the corset. We notice that this information may have been subject to information bias from patients. The lumbosacral corset is fairly tolerated as it has no side effects apart from minor discomfort or skin irritation (8). As observed by other 
authors, patients actually approve of lumbosacral corsets, hence the good compliance (8).

This study had several limitations. It depended on patients' information about days off duty and compliance to use of the lumbosacral corset. This could have suffered from recall or information bias. We also did not stratify patients on other confounders of NSALBP such as type of mattress used and body mass index. Although randomization was supposed to cater for these, they remain as our main limitations. The study however draws strength in being the first to examine the effect of the lumbosacral corset among patients with NSALBP.

\section{Conclusion}

We have demonstrated that the use of lumbosacral corset in addition to analgesic protocol among patients with NSALBP results in a significant improvement in their back specific disability compared to use of analgesia alone. The precise mechanism by which this occurs should be the subject of future trials. Future trials should also control for various confounders and be done across multiple centers.

\section{References}

1. Calmels P, Queneau P, Hamonet C et al. Effectiveness of a Lumbar Belt in Subacute Low Back Pain. Spine. 2009; 34(3):215-20

2. McCarberg B. Acute Back Pain: Benefits and Risks of Current Treatments. Curr Med Res Opin. 2010;26(1):179-90

3. Jellema P, Van Tulder MW, Van Poppel M et al. Lumbar Supports for Prevention and Treatment of Low Back Pain. Spine (Phila Pa 1976). 2001; 26(4):377-86.
4. Van Poppel MN, de Looze MP, Koes BW et al. Mechanisms of action of lumbar supports: a systematic review. Spine (Phila Pa 1976). 2000;25(16):2103-13.

5. Van Duijvenbode I, Jellema P, van Poppel M et al. Lumbar Supports for Prevention and Treatment of Low Back Pain. Cochrane Database Syst Rev. 2008; 2.

6. Fairbank JC, Pynsent PB. The Oswestry Disability Index. Spine (Phila Pa 1976). 2000; 25(22):2940-52

7. Costa LCM, Maher CG, Hancock MJ et al. Prognosis of Acute and Persistent Low Back Pain. CMAJ 2012; 184(11): 613-24

8. Valle-Jones JC, Walsh H, O'hara J et al. Controlled Trial of a Back Support ('Lumbotrain') in Patients with Non-specific Low Back Pain. Curr Med Res Opin 1992;12(9):604-13.

9. Steele J, Bruce-Low S, Smith D. A Reappraisal of the Deconditioning Hypothesis in Low Back Pain: Review of Evidence From a Triumvirate of Research Methods on Specific Lumbar Extensor Deconditioning. Curr Med Res Opin. 2014;30(5):865-911.

10. Mohamedali S, Mutiso SK, Oroko P et al. Effect of Additional Ultrasound to Analgesics in Treatment of Acute Low Back pain: A Randomized Controlled Trial Conducted at The Aga Khan Univeristy Hospital, Nairobi (AKUH,N). SA Orthopaedic Journal. 2014; 13(3):64-9.

11. Fairbank JC, Pynsent PB. The Oswestry Disability Index. Spine (Phila Pa 1976). 2000; 25(22):2940-52 